\title{
The Risk of Asthma in Patients with Ankylosing Spondylitis: A Population-Based Cohort Study
}

\author{
Te-Chun Shen ${ }^{1,2}$, Cheng-Li Lin ${ }^{3}$, Chang-Ching Wei ${ }^{4}$, Chia-Hung Chen ${ }^{1,2}$, Chih-Yen Tu ${ }^{2 *}$, \\ Te-Chun $\mathrm{Hsia}^{2}$, Chuen-Ming Shih ${ }^{2}$, Wu-Huei Hsu' ${ }^{2}$, Fung-Chang Sung ${ }^{1,3 * \neq}$ \\ 1 Graduate Institute of Clinical Medicine Science, College of Medicine, China Medical University, Taichung, \\ Taiwan, 2 Division of Pulmonary and Critical Care Medicine, Department of Internal Medicine, China Medical \\ University Hospital, China Medical University, Taichung, Taiwan, 3 Management Office for Health Data, \\ China Medical University Hospital, China Medical University, Taichung, Taiwan, 4 Division of Nephrology, \\ Department of Pediatrics, China Medical University Hospital, China Medical University, Taichung, Taiwan \\ $\ddagger$ These authors contributed equally to this work. \\ * fcsung.tw@gmail.com (F-CS); chesttu@gmail.com (C-YT)
}

\section{G open AcCess}

Citation: Shen T-C, Lin C-L, Wei C-C, Chen C-H, Tu C-Y, Hsia T-C, et al. (2015) The Risk of Asthma in Patients with Ankylosing Spondylitis: A PopulationBased Cohort Study. PLoS ONE 10(2): e0116608. doi:10.1371/journal.pone. 0116608

Academic Editor: James T. Rosenbaum, Oregon Health \&amp; Science University, UNITED STATES

Received: July 9, 2014

Accepted: December 11, 2014

Published: February 6, 2015

Copyright: @ 2015 Shen et al. This is an open access article distributed under the terms of the Creative Commons Attribution License, which permits unrestricted use, distribution, and reproduction in any medium, provided the original author and source are credited.

Data Availability Statement: The authors do not own the data underlying the study. The study population's data were from Taiwan NHIRD (http://w3. nhri.org.tw/nhird//date_01.html) and are maintained by Taiwan National Health Research Institutes (http:// nhird.nhri.org.tw/). The National Health Research Institutes (NHRI) is a non-profit foundation established by the government. The data is freely available to interested researchers from the NHRI upon request.

Funding: This study is supported in part by Taiwan Ministry of Health and Welfare Clinical Trial and Research Center of Excellence (MOHW103-TDU-B-

\section{Abstract}

\section{Background}

The relationship between asthma and ankylosing spondylitis (AS) is controversial. We examined the risk of asthma among AS patients in a nationwide population.

\section{Methods}

We conducted a retrospective cohort study using data from the National Health Insurance (NHI) system of Taiwan. The cohort included 5,974 patients newly diagnosed with AS from 2000 to 2010. The date of diagnosis was defined as the index date. A 4-fold of general population without AS was randomly selected frequency matched by age, gender and the index year. The occurrence and hazard ratio (HR) of asthma were estimated by the end of 2011.

\section{Results}

The overall incidence of asthma was 1.74 folds greater in the AS cohort than in the non-AS cohort (8.26 versus 4.74 per 1000 person-years) with a multivariable Cox method measured adjusted HR of 1.54 (95\% confidence interval $(\mathrm{Cl}), 1.34-1.76)$. The adjusted HR of asthma associated with AS was higher in women $(1.59 ; 95 \% \mathrm{Cl}, 1.33-1.90)$, those aged $50-64$ years $(1.66 ; 95 \% \mathrm{Cl}, 1.31-2.09)$, or those without comorbidities $(1.82 ; 95 \% \mathrm{Cl}, 1.54-2.13)$.

\section{Conclusion}

Patients with AS are at a higher risk of developing asthma than the general population, regardless of gender and age. The pathophysiology needs further investigation. 
212-113002), China Medical University Hospital, Academia Sinica Taiwan Biobank, Stroke Biosignature Project (BM103010096), NRPB Stroke Clinical Trial Consortium (MOST 103-2325-B-039006), Tseng-Lien Lin Foundation, Taichung, Taiwan, Taiwan Brain Disease Foundation, Taipei, Taiwan, and Katsuzo and Kiyo Aoshima Memorial Funds, Japan. The funders had no role in study design, data collection and analysis, decision to publish, or preparation of the manuscript.

Competing Interests: The authors have declared that no competing interests exist.

\section{Introduction}

Spondyloarthropathies (SpAs) are a group of interrelated diseases characterized by joint inflammation and extra-articular involvement, including mainly the axial and peripheral type and extra-articular type [1-3]. Ankylosing spondylitis (AS) is an axial and peripheral type of $\mathrm{SpA}$, which mainly affects the joints of the axial skeleton; however, extra-articular features may also occur. AS can affect the tracheobronchial tree and pulmonary parenchyma and is associated with several pulmonary manifestations [4-12]. Recent studies reported in the United States that the overall age-adjusted prevalence of SpA ranged from $0.9 \%-1.4 \%$ based on the diagnosis criteria, with the prevalence rates similar in men and women $[12,13]$. Another epidemiologic study has reported a prevalence of 16.7 per 10,000 in Asian population [14].

Asthma is a chronic inflammatory disorder of the airways and it is a representative disease of atopic disorders. Rudwaleit $e t$ al have reported that the prevalence rates of atopic disorders were 61/248 (24.6\%) in AS patients and 111/536 (20.7\%) in the control population, while the prevalence rates of asthma were 18/248 (7.3\%) in AS patients and 35/536 (6.5\%) in the control population. They concluded that there was a slight but insignificant increase in atopic disorders in AS patients [15]. However, this study used a questionnaire-based survey involving a small study population. In two nationwide population-based cohort studies of ours, an increased risk of asthma was identified in patients with systemic lupus erythematosus (SLE) and rheumatoid arthritis (RA) [16-17]. AS is thought to have an autoimmune component, we suggest that AS may be associated with asthma occurrence.

Autoimmune diseases (ADs) are caused by the loss of immune tolerance and are characterized by $\mathrm{T}$ - or B-cell activation leading to tissue damage. Patients with $\mathrm{AD}$ can be defined by a direct and indirect circumstantial evidence. Because of lack of the direct proof, patients with AS have limited features of AD [18]. However, patients with AS also exhibits the features of autoinflammation with activated innate immune system, which may appear at specific diseaseprone sites and be determined by local factors [19]. However, a definitive relationship between AS and asthma remains unclear.

This study attempts to determine whether patients with AS are at an elevated risk of developing asthma. We conducted a retrospective cohort study using nationwide population-based data, the National Health Insurance Research Database (NHIRD) of Taiwan.

\section{Materials and Methods \\ Data Source}

The National Health Insurance (NHI) program in Taiwan was launched in 1995 to provide comprehensive medical care for over $99 \%$ of the 23.74 million people residing in Taiwan (http://www.nhi.gov.tw/english/index.aspx). The NHIRD, established by the Bureau of National Health Insurance (BNHI) and the National Health Research Institutes (NHRI), is one of the large administrative health care databases in the world and is available to scientists for research purposes. All identification numbers of insured people and healthcare facilities in this database are encrypted for privacy protection. The encrypted identification numbers are unique, enabling record linking. The present study used the electronic data of the Longitudinal Health Insurance Database (LHID) obtained from NHRI, consisting of claims data of $1,000,000$ individuals randomly sampled from all population. The data can be retrospectively traced from 1996 onwards and followed up to the end of 2011. The NHRI reported no statistically significant differences in gender, age, and health care cost distribution between these subjects and whole insured population. This study was exempted from full ethical review by the China Medical University and Hospital Research Ethics Committee (IRB permit number: 
CMU-REC-101-012). The diagnostic code is in the format of the International Classification of Disease, 9th Revision, Clinical Modification (ICD-9-CM).

\section{Sampled Participants}

We identified a cohort of patients newly diagnosed with AS (ICD-9-CM code: 720 and 720.0) $[20,21]$ between January 1, 2000 and December 31, 2010. The date of the initial diagnosis was defined as the index date. Patients with a history of asthma (ICD-9-CM code: 493) or those with missing age or gender information were excluded. For each AS case identified, four insured controls adhering to the same inclusion criteria were randomly selected into the non-AS cohort. The subjects were frequency-matched by age (within 5 year), gender, and index year to reduce confounding effects of age and gender and to reduce measure bias of follow-up.

\section{Outcome and relevant variables}

Each subject was monitored from the index date until a new diagnosis of asthma was reached or until the subject was eliminated because of failure of follow-up, death, withdrawal from the insurance system, or the end of follow-up on December 31,2011. The baseline history of comorbidity and medication for each subject was determined from the claims data, including rhinitis (ICD-9CM codes: 472.0 and 477), chronic sinusitis (ICD-9-CM code: 473), gastroesophageal reflux disease (GERD; ICD-9-CM codes: 530.11, 530.81), obstructive sleep apnea (OSA; ICD-9-CM codes: $327.23,780.51,780.53,780.57$ ), chronic obstructive pulmonary disease (COPD; ICD-9-CM codes: 496) and usage of aspirin and non-steroidal anti-inflammatory drugs (NSAIDs) [22, 23].

\section{Statistical analysis}

Distributions of categorical demographic factors including gender and age $(<20,20-34,35-49$, $50-64$ and $\geq 65$ years) and comorbidities including rhinitis, chronic sinusitis, GERD, OSA, COPD, and usage of aspirin and NSAIDs, were compared between the AS and non-AS cohorts. Differences were examined using the Chi-square test for categorical variables and the $t$-test for continuous variables. The incidence density rates by gender, age, and comorbidity for each cohort were estimated by follow-up time (in person-years). The AS cohort to non-AS cohort incidence rate ratio (IRR) with 95\% confidence intervals (CI) was determined using the Poisson regression model. The multivariable Cox proportional hazards regression model was used to assess the risk of asthma associated with AS, comparing with the non-AS cohort and adjusted for demographic factors, comorbidities of rhinitis, chronic sinusitis, GERD, OSA, COPD, and usage of aspirin and NSAIDs. We also used the Kaplan-Meier method to estimate the cumulative asthma incidence for the two cohorts in the follow-up period and examined the difference using the log-rank test. All analyses were performed using the SAS statistical package (version 9.3 for windows; SAS Institute, Cary, NC), with the statistical level of significance being 0.05 .

\section{Results}

The study cohorts consisted of 5,974 AS patients and 23,896 non-AS control subjects. The age and gender distributions were similar in both cohorts (Table 1). Most subjects were 20-49 years of age (53.7\%) in both cohorts. Mean ages of the AS and non-AS cohorts were $46.3 \pm 17.4$ and $45.9 \pm 17.7$ years, respectively. Comorbidities including rhinitis, chronic sinusitis and GERD, and usage of aspirin and NSAIDs were more prevalent in AS patients than controls (p-values, <0.001). The average follow-up period was $6.67 \pm 3.30$ years $(39,838$ person-years $)$ for the AS cohort and $6.72 \pm 3.27$ years $(160,617$ person-years) for the non-AS cohort. The Kaplan-Meier analysis showed that by the end of the 12-year follow-up period, the cumulative 
Table 1. Demographic characteristics, comorbidity and medication in patients with and without ankylosing spondylitis.

\begin{tabular}{|c|c|c|c|c|c|}
\hline \multirow[t]{3}{*}{ Variables } & \multicolumn{4}{|c|}{ Ankylosing spondylitis } & \multirow[t]{3}{*}{ p-value ${ }^{t}$} \\
\hline & \multicolumn{2}{|c|}{ No $(N=23,896)$} & \multicolumn{2}{|c|}{ Yes $(N=5,974)$} & \\
\hline & $\mathbf{n}$ & $\%$ & $\mathbf{n}$ & $\%$ & \\
\hline Sex & & & & & 0.99 \\
\hline Male & 12620 & 52.8 & 3155 & 52.8 & \\
\hline Female & 11276 & 47.2 & 2819 & 47.2 & \\
\hline Age, years & & & & & 0.99 \\
\hline$<20$ & 1233 & 5.16 & 308 & 5.16 & \\
\hline 20-34 & 5939 & 24.9 & 1485 & 24.9 & \\
\hline $35-49$ & 6888 & 28.8 & 1722 & 28.8 & \\
\hline $50-64$ & 5884 & 24.6 & 1471 & 24.6 & \\
\hline$\geq 65$ & 3952 & 16.5 & 988 & 16.5 & \\
\hline Mean $(S D)^{\dagger}$ & 45.9 & 17.7 & 46.3 & 17.4 & 0.13 \\
\hline \multicolumn{6}{|l|}{ Comorbidity } \\
\hline Rhinitis & 2938 & 12.3 & 1052 & 17.6 & $<0.001$ \\
\hline Chronic sinusitis & 381 & 1.59 & 152 & 2.71 & $<0.001$ \\
\hline GERD & 218 & 0.91 & 129 & 2.16 & $<0.001$ \\
\hline OSA & 56 & 0.23 & 34 & 0.57 & $<0.001$ \\
\hline COPD & 1294 & 5.42 & 449 & 7.52 & $<0.001$ \\
\hline \multicolumn{6}{|l|}{ Medication } \\
\hline Aspirin & 4795 & $(20.1)$ & 1493 & $(25.0)$ & $<0.001$ \\
\hline NSAIDs & 8929 & (37.4) & 4017 & (67.2) & $<0.001$ \\
\hline \multicolumn{6}{|l|}{$\begin{array}{l}\text { Chi-square test } \\
{ }^{\dagger} \text { Two sample t-tes }\end{array}$} \\
\hline
\end{tabular}

doi:10.1371/journal.pone.0116608.t001

probability of developing asthma was 3.7\% higher for the AS cohort than the non-AS cohort (log-rank test, $\mathrm{p}<0.001$; Fig. 1$)$.

Table 2 shows there were a total of 1,090 asthma events. The incidence was 1.74-fold (95\% $\mathrm{CI}=1.53-1.98)$ higher in the AS cohort than in the non-AS cohort (8.26 vs. 4.74 per 1000 person-years), with an adjusted hazard ratio (HR) of 1.54 (95\% CI $=1.34-1.76)$ after controlling for age, gender, comorbidities and medications. The incidence of asthma was higher in women than in men in both cohorts. The adjusted HR of asthma in men and women was 1.47 $(95 \% \mathrm{CI}=1.20-1.80)$ and $1.59(95 \% \mathrm{CI}=1.33-1.90)$, respectively. The incidence increased with age in both cohorts. The age-specific adjusted HRs for the AS cohort, compared with the non-AS cohort, were significant for all age groups, except the youngest age group. Among those without comorbidities, the AS patients had an even higher adjusted HR of 1.82 (95\% $\mathrm{CI}=1.54-2.13$ ) for asthma. Table 3 shows that comorbidities increased the adjusted HR of asthma to 2.49 (95\% CI $=1.99-3.12)$ for the AS cohort.

\section{Discussion}

To the best of our knowledge, this is the first nationwide population-based study evaluating the relationship between AS and subsequent risk of asthma. We identified a significant risk of asthma among AS patients compared to the general population. Sex-specific analysis shows the 


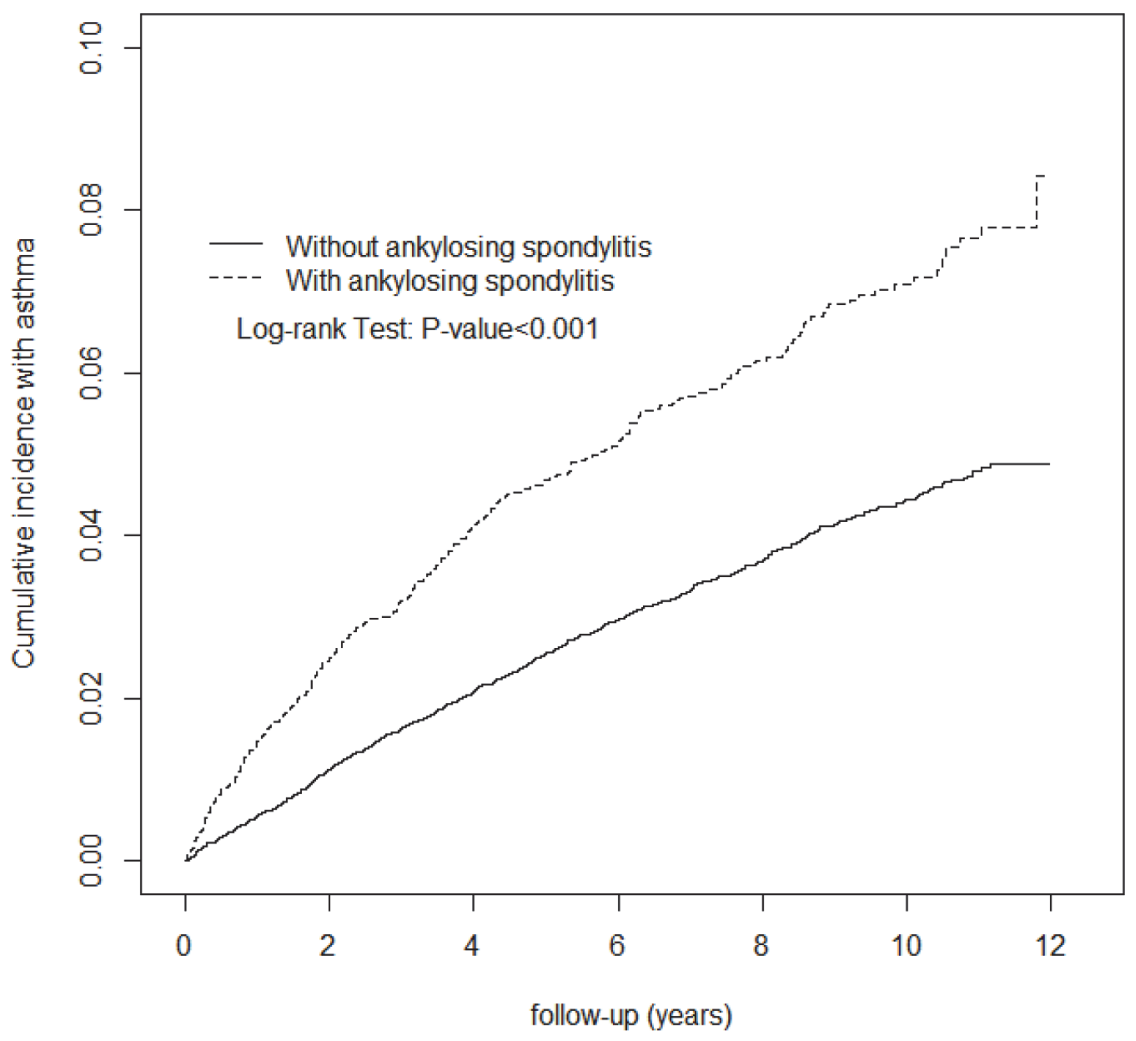

Fig 1. Cumulative incidence of asthma in patients with and without ankylosing spondylitis.

doi:10.1371/journal.pone.0116608.g001

highest asthmatic risk for females with AS patients. In addition, we found AS patients without comorbidities had a near 2-fold higher asthmatic risk than controls without comorbidities. Our data suggest that AS may play an independent role in the development of asthma.

The incidence of asthma increased with age in both cohorts. However, the crude AS patients to controls HR was the highest in the 30-49 years. The possible explanation for this might be that older subjects in both cohorts tend to be more prevalent with comorbidities, which may account for late-onset asthma. Risk factors may also include occupational exposure to irritants, environmental pollutants, female sex hormones, upper airway diseases, medications, respiratory infections, obesity, and stressful life events [24]. The crude HR of the older age groups was therefore lower than the younger age groups.

Few studies have discussed the relationship between AS and risk of asthma. Rudwaleit et al. reported the prevalence of asthma to be $7.3 \%$ in AS patients and $6.5 \%$ in a control population [15]. They used a questionnaire-based method and concluded that there is a slight and insignificant increase in asthma in AS patients. The diagnosis of asthma in AS patients is a challenge for physicians because pulmonary function tests are often influenced by chest wall restriction. Dincer et al. have reported that AS patients may experience deteriorated pulmonary function: the forced expiratory volume during the first second (FEV1) and FEV1 to forced vital capacity (FVC) ratio are decreased in AS patients as compared with controls [25]. Therefore, the diagnosis of asthma should be based on characteristic symptom patterns and evidence of variable airflow limitation from the bronchodilator reversibility or bronchial provocation tests, rather than from absolute values obtained from pulmonary function tests.

Several studies have shown a decreased risk of atopic diseases in patients with autoimmune diseases such as RA [15, 26-27]. However, these studies were primarily either only 
Table 2. Incidence of asthma and Cox method estimated ankylosing spondylitis cohort to non-ankylosing spondylitis cohort hazard ratio.

\begin{tabular}{|c|c|c|c|c|c|c|c|c|}
\hline \multirow[t]{3}{*}{ Variables } & \multicolumn{6}{|c|}{ Ankylosing spondylitis } & \multirow[t]{3}{*}{ Crude $\mathrm{HR}^{\S}(95 \% \mathrm{Cl})$} & \multirow[t]{3}{*}{ Adjusted $\mathrm{HR}^{\dagger}(95 \% \mathrm{Cl})$} \\
\hline & \multicolumn{3}{|c|}{ No $(\mathrm{N}=23,896)$} & \multicolumn{3}{|c|}{ Yes $(N=5,974)$} & & \\
\hline & $\mathbf{n}$ & Person-years & rate $^{\#}$ & $\mathbf{n}$ & Person-years & rate $^{\#}$ & & \\
\hline Total & 761 & 160617 & 4.74 & 329 & 39838 & 8.26 & $1.74(1.53,1.98)^{* * *}$ & $1.54(1.34,1.76)^{* * *}$ \\
\hline \multicolumn{9}{|l|}{ Sex } \\
\hline Male & 341 & 84939 & 4.01 & 141 & 21186 & 6.66 & $1.66(1.36,2.02)^{* * *}$ & $1.47(1.20,1.80)^{* * *}$ \\
\hline Female & 420 & 75677 & 5.55 & 188 & 18652 & 10.1 & $1.81(1.53,2.15)^{* * *}$ & $1.59(1.33,1.90)^{* * *}$ \\
\hline \multicolumn{9}{|l|}{ Age, years } \\
\hline$<34$ & 131 & 48936 & 2.68 & 51 & 12468 & 4.09 & $1.53(1.11,2.12)^{* * *}$ & $1.24(0.88,1.75)$ \\
\hline $35-49$ & 176 & 49246 & 3.57 & 86 & 12194 & 7.05 & $1.97(1.52,2.55)^{* * *}$ & $1.55(1.18,2.03)^{* * *}$ \\
\hline $50-64$ & 237 & 39881 & 5.94 & 108 & 9651 & 11.2 & $1.88(1.50,2.36)^{* * *}$ & $1.66(1.31,2.09)^{* * *}$ \\
\hline$\geq 65$ & 217 & 22553 & 9.62 & 84 & 5524 & 15.2 & $1.57(1.22,2.03)^{* * *}$ & $1.51(1.17,1.95)^{* *}$ \\
\hline \multicolumn{9}{|l|}{ Comorbidity } \\
\hline None $^{\ddagger}$ & 517 & 137393 & 3.76 & 233 & 31558 & 7.38 & $1.97(1.69,2.30)^{* * *}$ & $1.82(1.54,2.13)^{* * *}$ \\
\hline Rhinitis & 128 & 16397 & 7.81 & 55 & 5898 & 9.33 & $1.19(0.87,1.64)$ & $1.17(0.85,1.60)$ \\
\hline Chronic sinusitis & 20 & 2179 & 9.18 & 15 & 869 & 17.3 & $1.84(0.94,3.60)$ & $1.79(0.91,3.51)$ \\
\hline GERD & 3 & 732 & 4.10 & 4 & 423 & 9.47 & $2.20(0.49,9.84)$ & $2.39(0.52,10.9)$ \\
\hline OSA & 0 & 232 & 0.00 & 0 & 142 & 0.00 & - & - \\
\hline COPD & 143 & 7172 & 19.9 & 44 & 2622 & 17.8 & $0.85(0.61,1.19)$ & $0.86(0.61,1.21)$ \\
\hline \multicolumn{9}{|l|}{ Medication } \\
\hline \multicolumn{9}{|l|}{ Aspirin } \\
\hline No & 565 & 132646 & 4.26 & 242 & 30937 & 7.82 & $1.84(1.58,2.14)^{* * *}$ & $1.62(1.39,1.89)^{* * *}$ \\
\hline Yes & 196 & 27971 & 7.01 & 87 & 8902 & 9.77 & $1.40(1.09,1.80)^{* *}$ & $1.32(1.02,1.71)^{*}$ \\
\hline \multicolumn{9}{|l|}{ NSAIDs } \\
\hline No & 389 & 108914 & 3.57 & 89 & 14935 & 5.96 & $1.68(1.33,2.11)^{* * *}$ & $1.60(1.27,2.02)^{* * *}$ \\
\hline Yes & 372 & 51703 & 7.19 & 240 & 24903 & 9.64 & $1.36(1.16,1.60)^{* * *}$ & $1.48(1.26,1.74)^{* * *}$ \\
\hline
\end{tabular}

Rate $^{\#}$ per 1000 person-years

Crude $\mathrm{HR}^{\S}$, relative hazard ratio

${ }^{\dagger}$ Model was adjusted for age and comorbidities of rhinitis, chronic sinusitis, GERD, OSA, COPD and usage of aspirin and NSAIDs

‡ Patients without any one of the comorbidities (rhinitis, chronic sinusitis, GERD, OSA, and COPD) were classified as the none group

${ }^{*} \mathrm{p}<0.05,{ }^{*} \mathrm{p}<0.01,{ }^{*} * \mathrm{p}<0.001$

doi:10.1371/journal.pone.0116608.t002

Table 3. Cox method estimated hazard ratios of asthma associated ankylosing spondylitis and comorbidity.

\begin{tabular}{lllll}
\hline \multicolumn{1}{c}{ Variables } & & & Event & Adjusted HR $^{\dagger}$ (95\%Cl) \\
\hline Ankylosing spondylitis & Comorbidity ${ }^{\ddagger}$ & $\mathrm{N}$ & $\mathrm{n}$ & p-value $^{\#}$ \\
No & No & 19716 & 517 & 1(Reference) \\
No & Yes & 4180 & 244 & $2.31(1.98,2.70)^{* * *}$ \\
Yes & No & 4493 & 233 & $1.83(1.56,2.14)^{* * *}$ \\
Yes & Yes & 1481 & 96 & $2.49(1.99,3.12)^{* * *}$ \\
\hline
\end{tabular}

${ }^{\dagger}$ Model was adjusted for age, sex and usage of aspirin and NSAIDs

"p-value for interaction

${ }^{\ddagger}$ Patients with any one of the comorbidities of rhinitis, chronic sinusitis, GERD, OSA, and COPD were classified as the comorbidity group

***p $<0.001$

doi:10.1371/journal.pone.0116608.t003 
questionnaire based or using a small study population. Most researchers assumed that the establishment of an atopic phenotype reduces the risk of the subsequent development of autoimmune diseases. AS has both autoimmune component and autoinflammatory component. An early study has shown that AS is associated with HLA-B27 which may modulate the inflammatory response via misfolding with an unfolded protein response and/or via antigen recognition. Diseases associated with HLA-B27 are more likely autoinflammatory than autoimmune in nature [28]. However, the causality between AS and asthma remains largely unknown.

There are a number of factors that influence the risk of developing asthma. Although asth$\mathrm{ma}$ is a representative atopic disorder, the pathophysiological mechanisms are very complex. Development of asthma can be attributed to host factors such as genetics, obesity, and gender and environmental factors such as allergens, infections, occupational exposure, tobacco smoke, air pollution, and diet. Additionally, racial and ethnic differences, lifestyle, socioeconomic status, and the efficacy of the public health system have been linked to the prevalence of asthma [14]. In the present study, non-atopic asthma may also play a role in AS patients. To summarize, the increased risk of asthma in AS patients does not reflect an association between atopic asthma and AS.

A major strength of our study is that it was performed using population-based data that are highly representative of the general population. However, certain limitations should be considered. First, this study used the ICD-9-CM algorithm to define AS, asthma and comorbidities. The diagnosis depends on the performance of clinical physicians. An ad hoc committee established by the insurance authority was in charge of evaluating the claims data to prevent errors and violation. In addition, we selected only diagnosis appeared at least twice within a year to increase the validity and accuracy of diagnosis. Second, information on smoking, diet, occupational exposure, and family disease history was not available in the data analysis for adjusting the association between AS and asthma. Third, relevant clinical data such as pulmonary function tests, laboratory data on urinary and blood tests, imaging results, and pathology findings of the subjects were also unavailable in our study. Therefore, the statistical quality in a retrospective cohort study may be weaker.

\section{Conclusion}

Our data suggest that patients with AS have a significantly higher risk of developing asthma than the general population regardless of gender and age. Although, the pathophysiology association between AS and asthma needs further investigation.

\section{Acknowledgments}

This study is supported in part by Taiwan Ministry of Health and Welfare Clinical Trial and Research Center of Excellence (MOHW103-TDU-B-212-113002), China Medical University Hospital, Academia Sinica Taiwan Biobank, Stroke Biosignature Project (BM103010096), NRPB Stroke Clinical Trial Consortium (MOST 103-2325-B-039-006), Tseng-Lien Lin Foundation, Taichung, Taiwan, Taiwan Brain Disease Foundation, Taipei, Taiwan, and Katsuzo and Kiyo Aoshima Memorial Funds, Japan. The funders had no role in study design, data collection and analysis, decision to publish, or preparation of the manuscript.

\section{Author Contributions}

Conceived and designed the experiments: TCS CCW. Performed the experiments: TCS CCW CLL CHC FCS CYT TCH CMS WHH. Analyzed the data: CLL CHC FCS. Contributed reagents/materials/analysis tools: TCS CCW CLL CHC FCS CYT TCH CMS WHH. Wrote the paper: TCS CLL CYT FCS. Administrative support: TCH CMS WHH. 


\section{References}

1. Reveille JD, Arnett FC (2005) Spondyloarthritis: update on pathogenesis and management. Am J Med 118: 592-603. PMID: 15922688

2. Rudwaleit M, van der Heijde D, Landewé R, Akkoc N, Brandt J, et al. (2011) The Assessment of SpondyloArthritis International Society classification criteria for peripheral spondyloarthritis and for spondyloarthritis in general. Ann Rheum Dis 70: 25-31. doi: 10.1136/ard.2010.133645 PMID: 21109520

3. Zeidler H, Amor B (2011) The Assessment in Spondyloarthritis International Society (ASAS) classification criteria for peripheral spondyloarthritis and for spondyloarthritis in general: the spondyloarthritis concept in progress. Ann Rheum Dis 70: 1-3. doi: 10.1136/ard.2010.135889 PMID: 21163805

4. Bulwark DW, Weissman DN, Doll NJ (1985) Pulmonary manifestations of rheumatic diseases. Clin Rev Allergy 3: 249-67. PMID: 3886120

5. Gupta SM, Johnston WH (1978) Apical pulmonary disease in ankylosing spondylitis. N Z Med J 88: 186-8. PMID: 280798

6. Hunninghake GW, Fauci AS (1979) Pulmonary involvement in the collagen vascular diseases. Am Rev Respir Dis 119: 471-503. PMID: 36018

7. Rumancik WM, Firooznia H, Davis MS Jr, Leitman BS, Golimbu C, et al. (1984) Fibrobullous disease of the upper lobes: an extraskeletal manifestation of ankylosing spondylitis. J Comput Tomogr 8: 225-9. PMID: 6744925

8. Tanoue LT (1992) Pulmonary involvement in collagen vascular disease: a review of the pulmonary manifestations of the Marfan syndrome, ankylosing spondylitis, Sjogren's syndrome and relapsing polychondritis. J Thorac Imaging 7: 62-77. PMID: 1578527

9. Wiedemann HP, Matthay RA (1989) Pulmonary manifestations of the collagen vascular diseases. Clin Chest Med 10: 677-722. PMID: 2689071

10. Wolson AH, Rohwedder JJ (1975) Upper lobe fibrosis in ankylosing spondylitis. Am J Roentgenol Radium Ther Nucl Med 124: 466-71. PMID: 1155685

11. Yuksekkaya R, Almus F, Celıkyay F, Celıkel S, Inanır A, et al. (2014) Pulmonary involvement in ankylosing spondylitis assessed by multidetector computed tomography. Pol J Radiol 79: 156-63. doi: 10 12659/PJR.889850 PMID: 24971158

12. Mercieca C, van der Horst-Bruinsma IE, Borg AA (2014) Pulmonary, renal and neurological comorbidities in patients with ankylosing spondylitis; implications for clinical practice. Curr Rheumatol Rep 16: 434. doi: 10.1007/s11926-014-0434-7 PMID: 24925589

13. Reveille JD, Witter JP, Weisman MH (2012) Prevalence of axial spondylarthritis in the United States: estimates from a cross-sectional survey. Arthritis Care Res 64: 905-10. doi: 10.1002/acr.21621 PMID: 22275150

14. Dean LE, Jones GT, MacDonald AG, Downham C, Sturrock RD, et al. (2014) Global prevalence of ankylosing spondylitis. Rheumatology 53: 650-7. doi: 10.1093/rheumatology/ket387 PMID: 24324212

15. Rudwaleit M, Andermann B, Alten R, Sörensen H, Listing J, et al. (2002) Atopic disorders in ankylosing spondylitis and rheumatoid arthritis. Ann Rheum Dis 61: 968-74. PMID: 12379517

16. Shen TC, Tu CY, Lin CL, Wei CC, Li YF (2014) Increased risk of asthma in patients with systemic lupus erythematosus. Am J Respir Crit Care Med 189: 496-9. doi: 10.1164/rccm.201310-1792LE PMID: 24528323

17. Shen TC, Lin CL, Wei CC, Tu CY, Li YF (2014) The risk of asthma in rheumatoid arthritis: a populationbased cohort study. QJM 107: 435-42. doi: 10.1093/qjmed/hcu008 PMID: 24448376

18. Pérez-Fernández OM, Mantilla RD, Cruz-Tapias $P$, Rodriguez-Rodriguez $A$, Rojas-Villarraga A, et al. (2012) Spondyloarthropathies in autoimmune diseases and vice versa. Autoimmune Dis 2012: 736384. doi: 10.1155/2012/736384 PMID: 22400103

19. McGonagle D, McDermott MF (2006) A proposed classification of the immunological diseases. PLoS Med 3: e297. PMID: 16942393

20. Chou CH, Lin MC, Peng CL, Wu YC, Sung FC, et al. (2014) A nationwide population-based retrospective cohort study: increased risk of acute coronary syndrome in patients with ankylosing spondylitis. Scand J Rheumatol 43: 132-6. doi: 10.3109/03009742.2013.822097 PMID: 24134400

21. Sun LM, Muo CH, Liang JA, Chang SN, Sung FC, et al. (2014) Increased risk of cancer for patients with ankylosing spondylitis: a nationwide population-based retrospective cohort study. Scand J Rheumatol 43: 301-6. doi: 10.3109/03009742.2013.863969 PMID: 24559186

22. Boulet LP, Boulay MÈ (2011) Asthma-related comorbidities. Expert Rev Respir Med 5: 377-93. doi: 10.1586/ers.11.34 PMID: 21702660 
23. Papaiwannou A, Zarogoulidis P, Porpodis K, Spyratos D, Kioumis I, et al. (2014) Asthma-chronic obstructive pulmonary disease overlap syndrome (ACOS): current literature review. J Thorac Dis 6 Suppl 1: S146-51. doi: 10.3978/j.issn.2072-1439.2014.03.04 PMID: 24672688

24. de Nijs SB, Venekamp LN, Bel EH (2013) Adult-onset asthma: is it really different? Eur Respir Rev 22: 44-52. doi: 10.1183/09059180.00007112 PMID: 23457164

25. Dincer U, Cakar E, Kiralp MZ, Bozkanat E, Kilac H, et al. (2007) The pulmonary involvement in rheumatic diseases: pulmonary effects of ankylosing spondylitis and its impact on functionality and quality of life. Tohoku J Exp Med 212: 423-30. PMID: 17660708

26. Kaptanoglu E, Akkurt I, Sahin O, Hocaoglu S, Nacitarhan V, et al. (2004) Prevalence of atopy in rheumatoid arthritis in Sivas, Turkey. A prospective clinical study. Rheumatol Int 24: 267-71. PMID: 14523571

27. Hajdarbegovic E, Thio B, Nijsten T (2014) Lower lifetime prevalence of atopy in rheumatoid arthritis. Rheumatol Int 34: 847-8. doi: 10.1007/s00296-013-2785-1 PMID: 23708584

28. Pham T (2008) Pathophysiology of ankylosing spondylitis: what's new? Joint Bone Spine 75: 656-60. doi: 10.1016/j.jbspin.2008.09.003 PMID: 19036625 